\title{
Common Variable Immunodeficiency and Its Inflammatory Neurological Manifestations: A Case Report and Literature Review
}

Bárbara Martins ( $\square$ barbarapmartins6@gmail.com)

Centro Hospitalar de São João EPE: Centro Hospitalar Universitario de Sao Joao https://orcid.org/0000-0003-3026-3453

Joana Miranda

Maria João Pinto

Andreia Costa

André Silva-Pinto

Natália Melo

Filipa Ceia

José Torres Costa

Joana Guimarães

\section{Research Article}

Keywords: Common variable immunodeficiency, neurological disease, autoimmunity, Granulomatous-lymphocytic interstitial lung disease

Posted Date: January 31st, 2022

DOI: https://doi.org/10.21203/rs.3.rs-1179009/v1

License: (c) (1) This work is licensed under a Creative Commons Attribution 4.0 International License. Read Full License 


\section{Abstract \\ Purpose}

Common variable immunodeficiency disorders (CVID) are a group of primary immunodeficiencies characterized by impaired immunoglobulin production and dysregulated immune response. Neurological manifestations have been described in few patients, and little is known about its clinic and therapeutic approach. Thus, the aim of this work was to review the literature on it and to help differentiate CVID from its mimics, especially sarcoidosis.

\section{Methods}

We described a case report and included a literature review of inflammatory neurological involvement in CVID.

\section{Results}

A 32-year-old female patient with a medical history of recurrent bacterial infections, temporal focal epilepsy and granulomatous lung disease under study, and cervix squamous cell carcinoma, was initially admitted to the emergency department due to intracranial hypertension. After excluding infectious and neoplastic etiologies, the most likely hypothesis was that granulomatous pulmonary, cerebral, and leptomeningeal inflammatory involvement were associated with sarcoidosis. Two years later, a diagnosis of CVID was made, and the patient was secondarily diagnosed with Granulomatous and Lymphocytic Interstitial Lung Disease (GLILD) and related inflammatory brain disease - both complications of CVID. After starting targeted treatment with immunoglobulin replacement and pulse glucocorticoids followed by a chronic taper, the patient became stable. However, three consecutive failures in immunoglobulin intake led to disease recurrence with relapse of neurological manifestations.

\section{Conclusion}

This case illustrates the complex multiple organ manifestations of CVID. When granulomatous conditions arise in these patients, GLILD with multisystem involvement should be taken in consideration. Early treatment with combined steroids and immunotherapy seems to be effective in controlling CVID's neurological manifestations.

\section{Introduction}

Common variable immunodeficiency disorders (CVID) are the most clinically significant primary immunodeficiency in adults, accounting for $90 \%$ of symptomatic primary antibody deficiencies [1]. They are characterized by low levels of serum immunoglobulin (Ig) G with IgA and/or IgM deficit due to a primary antibody failure [2]. Current estimates suggest a prevalence ranging from 1:25,000 to 1:100,000 in Caucasian individuals [1, 3]; recently, a Finnish study suggested a higher prevalence of 6.9:100,000 [3]. By definition, the causes of CVID are unknown, but an increasing number of monogenic causes and a digenic disorder have been described in patients with a CVID phenotype. Indeed, a causative variant, for example in NFKB1, NFKB2, CTLA-4, TCF3 genes, has been identified in approximately $25 \%$ of the cases in non-consanguineous populations [1]. Although commonly associated with recurrent bacterial infections, other systemic conditions such as autoimmune manifestations, inflammatory disorders and malignancy have been described [4]. Approximately $20 \%$ of these patients develop at least one autoimmune disease, frequently autoimmune thrombocytopenia, autoimmune hemolytic anemia, pernicious anemia, and rheumatoid arthritis [1]. Neurologic dysfunction in CVID is less known but can be associated with significant morbidity and mortality [5]. Thus, starting from a challenging clinical case, the aim of this work was to review the literature on the neurological manifestations of CVID and to help differentiate CVID from its mimics, especially sarcoidosis.

\section{Methods}

We present a CVID patient with significant neurologic morbidity and report an extensive literature review on inflammatory neurological involvement in this disease. Articles written in English, searched in PUBMED and academic google, using the Key Words "common variable immunodeficiency", "granulomatous disease" and "neurological manifestations" were included. We extracted data from case series, observational studies, systematic or no systematic reviews, and case reports.

\section{Results}

A 32-year-old Caucasian woman was admitted to the emergency department in April 2015 due to a holocranial headache with 2 weeks evolution of increasing intensity until $9 / 10$ in numerical pain scale, worsened by lying down. Additionally, vomiting, fever, and gait imbalance were reported. She had a past medical history of recurrent respiratory bacterial infections requiring antibiotics, adult-onset (30 years old) temporal focal epilepsy of unknown etiology (initially medicated with sodium valproate $1000 \mathrm{mg} /$ day, which was suspended in 2016, and later levetiracetam 1000 mg/day, suspended in 2020), granulomatous lung disease under study since 2013 at another institution (suspected sarcoidosis), and squamous cell carcinoma in situ of the uterine cervix. She had a family history of early onset neoplasms (gastrointestinal, breast, and ovary); with no consanguinity. On admission, she presented papilledema and right hemiataxia. A contrast brain CT showed at least two enhancing lesions in the right cerebellar hemisphere with a possible dural involvement, with marked perilesional edema, obstructive hydrocephalus, and brainstem compression (figure 1). The clinical picture was compatible with intracranial hypertension. Dexamethasone $5 \mathrm{mg}$ was urgently started, with symptom improvement, and the patient was admitted to an intensive care unit. The serological study showed 
low total protein levels $(55.0 \mathrm{~g} / \mathrm{L}$, normal range, NR, of $64-83 \mathrm{~g} / \mathrm{L})$ and low serum lgG (409 mg/dL, NR=520-1.560 mg/dL). Full blood count, erythrocyte sedimentation rate, vitamin B12 levels, thyroid function, autoimmune study [including rheumatoid factor, antinuclear antibody (ANA), antineutrophil cytoplasmic antibody (ANCA), anti-double strand DNA antibodies (anti-dsDNA), anti-Ro (SS-A), and anti-La (SS-B), anti-cardiolipin, anti-ovarian, and antineuronal antibodies], serum angiotensin-converting-enzyme (ACE) and tumor markers [carcinoembryonic antigen, carbohydrate antigen (Ca) 19-9, Ca 125] were normal/negative. Antibodies against myelin-oligodendrocyte-glycoprotein (Anti-MOG) and aquaporin 4 (Anti-AQ4) were not detected. Serum polymerase chain reaction (PCR) for varicella-zoster (VZV) and herpes simplex (HSV) 1 and 2, Treponema pallidum particle agglutination (TPPA), cytomegalovirus (CMV), Epstein-Barr virus (EBV), human immunodeficiency virus (HIV), Toxoplasma gondii, and Borrelia burgdorferis serology were negative. CSF analysis showed mild pleocytosis ( 6 cells/ $\mu \mathrm{l}$ ) with lymphocyte prevalence (99\%), normal proteins $(0.29 \mathrm{mg} / \mathrm{L} ; \mathrm{NR}=0.18-0.45 \mathrm{mg} / \mathrm{L})$, no glucose consumption, and normal ACE. CSF cytology was negative for malignancy; CSF bacterial, viral, and fungal workup was also negative. Brain MRI (April 28) revealed signs of cortico-pial infiltration at the level of both cerebellar hemispheres and right inferior temporal region, associated with edema, leading to left fourth ventricle compression and enlargement of the supratentorial ventricular system, with incipient signs of CSF transudation (figure 1, panel B) - suggesting an inflammatory process of granulomatous and/or infectious etiology, not excluding secondary neoplasia. Thoracic-abdominopelvic CT showed architectural distortion of the lung parenchyma, interlobular septal thickening, and linear and nodular densifications, mainly at the subpleural level and at the basal lung, with cylindrical bronchiectasis and several bilateral micronodules; normal spleen size in the upper limit of normality. Bronchoscopy revealed a normal total and differential cell count, absence of malignant cells, and negative microbiological study in bronchoalveolar lavage. A CT-guided lung biopsy showed lung parenchyma extensively occupied by small lymphoid cell infiltrates and areas of granulomatous outline, with rare giant cells without necrosis. The test for acid-resistant bacilli was negative. Pulmonary function tests were normal. Upper and lower gastrointestinal endoscopy, and cervical and breast ultrasound displayed no alterations. During hospitalization, she continued dexamethasone $4 \mathrm{mg}$ three times/day with progressive symptom improvement. She remained apyretic since admission. Ten days after starting glucocorticoid therapy (May 7), a second brain MRI found a clear reduction in the corticopial enhancement areas in the right cerebellar hemisphere, and no enhancement in the remaining areas, with a resolution of ventricular dilatation (figure 1, panel C). The patient was discharged with the diagnosis of sarcoidosis with pulmonary and neurological involvement, with tapering steroid therapy (initially $1 \mathrm{mg} / \mathrm{Kg} / \mathrm{day}$, tapering within 4-6 weeks to $20 \mathrm{mg}$ /day). Her symptoms and brain MRI abnormalities resolved within 3 months.

After this neurological presentation, CVID and granulomatous lung disease secondary to immunodeficiency (Granulomatous and Lymphocytic Interstitial Lung Disease - GLILD) diagnosis was made. Diagnostic work-up showed a low level of total lgG (380 mg/dL, NR<650 mg/dL), lgA (30 mg/dL, NR=47-206 mg/dL), $\operatorname{lgM}(47 \mathrm{mg} / \mathrm{dL})$ and $\lg E(<2 \mathrm{kU} / \mathrm{L})$, as well as lymphopenia, defective response to pneumonia vaccine (Pneumovax $\left.{ }^{\circledR} 23\right)$ antigens, decreased switched memory B cells (7.9\% of IgD-CD27+ B cells) and increased CD21low B cells (21.3\%). Cellular immune response was also affected, with inversion of the $\left(\mathrm{CD}^{+}\right) \mathrm{CD} 4^{+} / \mathrm{CD} 8^{+}$ratio, low naïve CD4+ T cells $(24 \% \mathrm{CD} 4+\mathrm{CD} 45 \mathrm{RA}+$ cells) and increased CD $4+$ memory $\mathrm{T}$ cells $(72 \% \mathrm{CD} 4+\mathrm{CD} 45+\mathrm{RO}+\mathrm{cells})$. The patient was started on IgIV $400 \mathrm{mg} / \mathrm{kg}$ every three weeks (added to low-dose oral steroid therapy - at that moment with $10 \mathrm{mg} /$ day of prednisolone).

She remained in clinical and imaging remission until June/2020 (figure 2), when, after missing three doses of IVIg during the COVID-19 pandemic, she complained of cervicalgia with occipital irradiation, refractory to analgesia. Brain CT showed a new hypodensity on the left cerebellar hemisphere, with a slight mass effect (figure 2), which motivated hospitalization. Cervical CT did not show any lesion. A new MRI confirmed cortico-pial enhancement in the cerebellar hemispheres and bilateral pons, and pachymeningeal enhancement in the cerebellar tent, with no restriction to diffusion (figure 2). MRI-spectroscopy did not suggest a malignant lesion. New CSF analysis revealed pleocytosis ( 112 cells $/ \mu \mathrm{l})$, with lymphocyte prevalence $(99 \%)$, and mildly elevated protein level $(0.78$ $\mathrm{mg} / \mathrm{L})$, glucose consumption (70\% of the serum value), normal ACE and Adenosine deaminase test (ADA), and negative bacteriological and mycobacteriological study. Oligoclonal bands were positive in CSF and negative in serum. Given the higher risk of CVID patients to develop lymphoproliferative diseases, cytological and imagiologic examinations were carried out to exclude CNS lymphoma. No malignant cells were detected in the CSF. A full-body 18-FDG Positron Emission Tomography (PET)/CT study revealed irregular densifications in both lungs (nodular and ground glass) and mediastinal-hilar bilateral adenopathies, with increased FDG-F18 uptake, and heterogeneous distribution of cerebellar FDG-F18 (figure 2). Based on these findings, a relapse of inflammatory brain disease associated with CVID was assumed. The patient underwent a cycle of high-dose steroid therapy (methylprednisolone $1 \mathrm{~g}$ for 5 days), followed by a maintenance dose of oral prednisolone $40 \mathrm{mg} /$ day, associated with trimethoprim-sulfamethoxazole three times/week and IVIg replacement therapy, as described above.

After 3 months, the patient was in clinical and imaging remission from a neurological point of view, with stability of the pulmonary radiographic findings (no respiratory symptoms). Currently, she keeps surveillance in neurology consultation every 3 months. The last clinical evaluation was on October/2021; she was asymptomatic, and the last brain MRI assessment in December/2021 showed no alterations.

\section{Discussion}

We report an uncommon and underreported manifestation of CVID: an inflammatory brain lesion in a patient with a lung granulomatous disease (GLILD).

According to the European Society for Immunodeficiencies (ESID), CVID patients are characterized by a family history of antibody deficiency and/or personal history of increased susceptibility to infection as well as autoimmune, granulomatous, or/and lymphoproliferative diseases [6, 7]. These clinical features combined with a marked decrease in IgG and IgA (with or without low IgM levels), and evidence B cell differentiation impairment allow the diagnosis of this disorder [1, 7]. Being a diagnosis of exclusion, transient and secondary causes of hypogammaglobulinemia, such as drugs (particularly rituximab and anticonvulsants) and profound T-cell deficiency should be first considered [1, 7]. In the present case, considering the clinical, imaging and disease progression, drug-related immunodeficiency was unlikely.

Although the most relevant cellular implication in CVID is related to B lymphocytes, it has been increasingly demonstrated that T cell impairment is a frequent and important finding in these patients. Our patient fulfills ESID diagnostic criteria for CVID, with additional evidence of decreased switched memory B cells, increased CD21 low cell numbers, and T cell population alterations (decreased naïve CD4+ T cell numbers as well as implications in the CD4+/CD8+ ratio). 
These findings are usually associated with the severity and poor outcome of CVID. Recent evidence indicates that lymphoproliferation and granulomatous diseases are mainly observed in patients with severe depletion of switched memory B cells and CD4+ naïve T cells (8). This subgroup of CVID patients is also characterized by an increased frequency of splenomegaly and malignancies [8].

Our patient had a diagnosis of a non-infectious inflammatory brain lesion secondary to CVID. Among the described cases in the literature (Table 1), infections are the main cause of CVID-associated neurological dysfunction, particularly meningitis and encephalitis [4, 9]. Autoimmune/inflammatory conditions comprise the second largest category of neurologic complications (1.4\% of CVID patients) [4,5], including myelitis [5, 9-12], brain inflammatory/demyelinating disease [4, 5, 7, 13], unilateral [2,5] and bilateral [14] optic neuritis, cerebral vasculitis [5], and encephalopathy (autoimmune encephalitis [5] and acute encephalomyelitis [15]). Van de Ven et al. [16] reported a 4\% prevalence of inflammatory CNS disease, higher than previously reported. Of the studies reporting inflammatory lesions, the majority are case reports [2, 4, 7, 9-15]; there are few observational studies [5, 16-18]. Although some of these studies report a higher frequency of neurological CVID manifestations in females [9, 17], in others [16], age and gender distribution were comparable between CVID controls without CNS manifestations and CNS disease patients (mean age $46.6 \mathrm{y} \pm 15$ in controls vs. $44.1 \mathrm{y} \pm 11.0$ in CNS disease). Overall, some authors suggested that CNS involvement in CVID is not an isolated manifestation but rather a symptom of severe systemic immune dysregulation, possibly due to failure of peripheral tolerance [2]. 
Table 1

Non-infectious inflammatory neurological manifestations of CVID

\begin{tabular}{|c|c|c|c|c|c|c|c|}
\hline $\begin{array}{l}\text { Author, } \\
\text { year }\end{array}$ & Type of study & $\begin{array}{l}\text { Gender, age, } \\
\text { clinical history }\end{array}$ & $\begin{array}{l}\text { CVID } \\
\text { diagnosis }\end{array}$ & $\begin{array}{l}\text { Neurological } \\
\text { manifestation }\end{array}$ & $\begin{array}{l}\text { Complementary } \\
\text { study }\end{array}$ & $\begin{array}{l}\text { Other CVID } \\
\text { manifestations }\end{array}$ & $\begin{array}{l}\text { Treatment ar } \\
\text { prognosis }\end{array}$ \\
\hline \multirow{4}{*}{$\begin{array}{l}\text { Kumar et } \\
\text { al., 2008 } \\
\text { [11] }\end{array}$} & \multirow[t]{4}{*}{ Case-report } & \multirow{4}{*}{$\begin{array}{l}\text { Female, 64y } \\
\text { Recurrent } \\
\text { infections and } \\
\text { idiopathic } \\
\text { thrombocytopenic } \\
\text { purpura, vitiligo, } \\
\text { psoriasis and } \\
\text { diabetes }\end{array}$} & \multirow[t]{4}{*}{ Yes } & \multirow[t]{4}{*}{ Myelitis } & CSF: normal & \multirow{4}{*}{$\begin{array}{l}\text { Pulmonary } \\
\text { sarcoidosis } \\
\text { suspected }\end{array}$} & \multirow{2}{*}{$\begin{array}{l}\text { Pulse glucoc } \\
\text { followed by c } \\
\text { taper+Inflixin }\end{array}$} \\
\hline & & & & & MRI spine: cervical & & \\
\hline & & & & & $\begin{array}{l}\text { hyperintensity with } \\
\text { contrast } \\
\text { enhancement }\end{array}$ & & \multirow{2}{*}{$\begin{array}{l}\text { Improvement } \\
\text { findings, } \\
\text { Persistent } \\
\text { neurological } \\
\text { symptoms }\end{array}$} \\
\hline & & & & & $\begin{array}{l}\text { MRI Brain: contrast } \\
\text { enhancement in the } \\
\text { right basal forebrain }\end{array}$ & & \\
\hline \multirow{4}{*}{$\begin{array}{l}\text { Sempere } \\
\text { et al., } \\
2011 \text { [14] }\end{array}$} & \multirow[t]{4}{*}{ Case-report } & Male, 26y & \multirow[t]{4}{*}{ Yes } & \multirow{4}{*}{$\begin{array}{l}\text { Bilateral optic } \\
\text { neuropathy }\end{array}$} & CSF: normal & & \multirow{4}{*}{$\begin{array}{l}\text { Methylpredni } \\
\text { IV and taper } \\
\text { prednisone + } \\
(0.4 \mathrm{~g} / \mathrm{kg} \mathrm{3-3} \\
\text { Asymptomat } \\
\text { mo }\end{array}$} \\
\hline & & $\begin{array}{l}\text { Hemolytic } \\
\text { anemia, }\end{array}$ & & & MRI Brain: mild & & \\
\hline & & $\begin{array}{l}\text { autoimmune } \\
\text { thrombocytopenia } \\
\text { and neutropenia }\end{array}$ & & & $\begin{array}{l}\text { enhancement of both } \\
\text { optic nerves without } \\
\text { other }\end{array}$ & & \\
\hline & & & & & abnormalities & & \\
\hline \multirow[t]{3}{*}{$\begin{array}{l}\text { Danieli et } \\
\text { al.,2012 } \\
\text { [12] }\end{array}$} & \multirow[t]{3}{*}{ Case-report } & \multirow[t]{3}{*}{ Female, 71y } & \multirow[t]{3}{*}{ Yes } & \multirow[t]{3}{*}{ Acute myelitis } & $\begin{array}{l}\text { MRI spine: cervical } \\
\text { and thoracic cord } \\
\text { hyperintensity with } \\
\text { contrast }\end{array}$ & & $\begin{array}{l}\text { Pulse glucoc } \\
\text { without chrol } \\
\text { glucocorticoi } \\
\text { Intramuscula }\end{array}$ \\
\hline & & & & & $\begin{array}{l}\text { C4 and T5-T10 } \\
\text { segments, mainly in } \\
\text { posterior columns }\end{array}$ & & $\begin{array}{l}\text { No new lesio } \\
\text { MRI after tre }\end{array}$ \\
\hline & & & & & MRI Brain: normal & & $\begin{array}{l}\text { Persistent } \\
\text { neurological } \\
\text { symptom }\end{array}$ \\
\hline \multirow{6}{*}{$\begin{array}{l}\text { Jabbari } \\
\text { et al., } \\
2014 \text { [10] }\end{array}$} & \multirow[t]{6}{*}{ Case-report } & Female, 41y & \multirow[t]{6}{*}{ Yes } & \multirow{6}{*}{$\begin{array}{l}\text { Longitudinally } \\
\text { extensive myelitis }\end{array}$} & $\begin{array}{l}\text { CSF: acelular, } 0.56 \\
\text { g/L protein neaative }\end{array}$ & & \multirow{6}{*}{$\begin{array}{l}\text { Pulse glucoc } \\
\text { without tapel } \\
\text { glucocorticoi } \\
\text { IVIG + Inpatiє } \\
\text { rehabilitation }\end{array}$} \\
\hline & & Recurrent & & & oligoclonal bands & & \\
\hline & & $\begin{array}{l}\text { Diffuse } \\
\text { adenopathy }\end{array}$ & & & $\begin{array}{l}\text { MRI spine: } \\
\text { lonqitudinally }\end{array}$ & & \\
\hline & & pyogenic & & & $\begin{array}{l}\text { extensive cord signal } \\
\text { T2 and contrast }\end{array}$ & & \\
\hline & & \multirow{2}{*}{$\begin{array}{l}\text { infections and } \\
\text { idiopathic } \\
\text { thrombocytopenic } \\
\text { purpura }\end{array}$} & & & $\begin{array}{l}\text { enhancement in } \\
\text { conus medullaris }\end{array}$ & & \\
\hline & & & & & MRI Brain: normal & & \\
\hline \multirow{5}{*}{$\begin{array}{l}\text { Nguyen } \\
\text { et al., } \\
2016 \text { [4] }\end{array}$} & \multirow[t]{5}{*}{ Case-report } & \multirow{5}{*}{$\begin{array}{l}\text { Female, 40y } \\
\text { Lymphocytic } \\
\text { colitis }\end{array}$} & \multirow[t]{5}{*}{ Yes, 2007} & \multirow{5}{*}{$\begin{array}{l}\text { 2009: unilateral } \\
\text { optic neuritis and } \\
\text { multifocal } \\
\text { choroidopathy } \\
\text { 2013: episodic } \\
\text { visual distortion } \\
\text { and headache } \\
\text { 2014: seizure }\end{array}$} & $\begin{array}{l}\text { CSF 2013: } \\
\text { lymphocytic }\end{array}$ & \multirow{5}{*}{$\begin{array}{l}\text { 2007: } \\
\text { Lymphocytic } \\
\text { interstitial } \\
\text { pneumonia } \\
\text { (LIP) }\end{array}$} & \multirow{5}{*}{$\begin{array}{l}\text { Pulse glucoc } \\
\text { without a tap } \\
\text { rituximab }+1 \text { ' } \\
\text { Since the adc } \\
\text { rituximab - r } \\
\text { neurologicall } \\
\text { with near-cor } \\
\text { resolution of } \\
\text { symptoms al } \\
\text { findings }\end{array}$} \\
\hline & & & & & & & \\
\hline & & & & & $\begin{array}{l}\text { Brain MRI: } 2 \\
\text { enhancing lesions } \\
\text { with mild mass effect }\end{array}$ & & \\
\hline & & & & & $\begin{array}{l}\text { in the cerebellum, } \\
\text { leptomeningeal } \\
\text { enhancement }\end{array}$ & & \\
\hline & & & & & $\begin{array}{l}\text { Brain biopsy }(2 x) \text { : } \\
\text { modest astrogliosis, } \\
\text { no granulomas }\end{array}$ & & \\
\hline \multirow[t]{4}{*}{$\begin{array}{l}\text { Abati et } \\
\text { al., } 2018 \\
\text { [2] }\end{array}$} & Case-report & Male, 26y & Yes & Optic neuropathy & $\begin{array}{l}\text { CSF: marked } \\
\text { pleocytosis ( } 200 \\
\text { cells/ul), }\end{array}$ & & $\begin{array}{l}\text { Course of IV } \\
\text { methylpredni }\end{array}$ \\
\hline & & & & & $\begin{array}{l}\text { mildly elevated } \\
\text { protein level }(56\end{array}$ & & and taper pre \\
\hline & & & & & $\mathrm{mg} / \mathrm{dl})$ & & Remained \\
\hline & & & & & $\begin{array}{l}\text { Brain MRI: } \\
\text { Enlargement and } \\
\text { hyperintensity of the } \\
\text { optic nerve in fat- } \\
\text { suppression }\end{array}$ & & follow-up \\
\hline
\end{tabular}




\begin{tabular}{|c|c|c|c|c|c|c|c|}
\hline $\begin{array}{l}\text { Author, } \\
\text { year }\end{array}$ & Type of study & $\begin{array}{l}\text { Gender, age, } \\
\text { clinical history }\end{array}$ & $\begin{array}{l}\text { CVID } \\
\text { diagnosis }\end{array}$ & $\begin{array}{l}\text { Neurological } \\
\text { manifestation }\end{array}$ & $\begin{array}{l}\text { Complementary } \\
\text { study }\end{array}$ & $\begin{array}{l}\text { Other CVID } \\
\text { manifestations }\end{array}$ & $\begin{array}{l}\text { Treatment ar } \\
\text { prognosis }\end{array}$ \\
\hline $\begin{array}{l}\text { Najem et } \\
\text { al.,2018 } \\
\text { [17] }\end{array}$ & $\begin{array}{l}\text { Observational and } \\
\text { retrospective study }\end{array}$ & $\mathrm{N}=19$ & $\begin{array}{l}8 \text { of the } 13 \\
\text { patients were } \\
\text { diagnosed } \\
\text { with CVID after } \\
\text { the diagnoses } \\
\text { of CNS } \\
\text { granulomas }\end{array}$ & $\begin{array}{l}68.4 \%(n=13) \\
\text { granulomas } \geq 2 \\
\text { organs including } \\
\text { CNS, 31.6\% } \\
\text { (6/19) CNS } \\
\text { granulomas only }\end{array}$ & $\begin{array}{l}\text { CSF ( } n=5) \text { : Increased } \\
\text { total proteins with/or } \\
\text { without lymphocytic } \\
\text { pleocytosis in } 80 \% \\
\text { High serum ACE in } \\
42.8 \%(3 / 7) \\
\text { MRI: brain mass } \\
\text { (70\%), } \\
\text { leptomeningeal } \\
\text { enhancement (10\%), } \\
\text { non-specific white } \\
\text { matter lesions (10\%) } \\
\text { and absence of } \\
\text { normal signal of } \\
\text { neurohypophysis } \\
\text { (10\%) }\end{array}$ & & $\begin{array}{l}26 \%(5 / 19) \mathrm{s} \\
\text { only, 26.3\% (! } \\
\text { steroids alon } \\
\text { immunoglob } \\
21 \%(4 / 19) \\
\text { immunoglob } \\
\text { only, } 15.8 \% \text { ( } \\
\text { infliximab, } 15 \\
\text { (3/19) other } \\
\text { (methotrexat } \\
\text { cyclosporine, } \\
\text { azathioprine, } \\
\text { adalimumab } \\
\text { cyclophosph } \\
\text { 10.5\% (2/19) } \\
\text { rituximab, an } \\
\text { (1/19) refuse } \\
\text { therapy }\end{array}$ \\
\hline $\begin{array}{l}\text { Farshad } \\
\text { et al., } \\
2019 \text { [9] }\end{array}$ & Case-report & $\begin{array}{l}\text { Female, 30y } \\
\text { Recurrent upper } \\
\text { respiratory } \\
\text { infections, vitiligo, } \\
\text { and immune } \\
\text { thrombocytopenic } \\
\text { purpura }\end{array}$ & Yes & $\begin{array}{l}\text { Transverse } \\
\text { myelitis }\end{array}$ & $\begin{array}{l}\text { Spinal MRI: signal } \\
\text { hyperintensity. Brain } \\
\text { MRI: hyperintensity in } \\
\text { the right middle } \\
\text { frontal gyrus }\end{array}$ & & $\begin{array}{l}\text { Chronic } \\
\text { glucocorticoi } \\
\text { without pulst } \\
\text { Methotrexat } \\
\text { Improvemen } \\
\text { findings } \\
\text { Persistent } \\
\text { neurological } \\
\text { symptoms in }\end{array}$ \\
\hline $\begin{array}{l}\text { Galli et } \\
\text { al., } 2019 \\
\text { [19] }\end{array}$ & $\begin{array}{l}\text { Case series } \\
\text { (University of Utah } \\
\text { Healthcare } \\
\text { system with CVID } \\
\text { and co-existing } \\
\text { epilepsy) }\end{array}$ & $\begin{array}{l}\mathrm{N}=5 \text { (3 male, } 2 \\
\text { female) } \\
\text { Mean Age Seizure } \\
\text { Onset: } 29 y(6.4) \\
\text { Mean Age CVID } \\
\text { Diagnosis: } 37 y \\
(22.8)\end{array}$ & Yes & $\begin{array}{l}\text { All patients had } \\
\text { atypical seizure: } \\
\text { behavioral arrest, } \\
\text { consciousness } \\
\text { alterations, } \\
\text { and/or } \\
\text { amnestic } \\
\text { episodes. } \\
\text { Generalized tonic } \\
\text { clonic seizures } \\
\text { less common. }\end{array}$ & $\begin{array}{l}\text { Most had abnormal } \\
\text { imaging findings, } \\
\text { most commonly } \\
\text { temporal lobe } \\
\text { asymmetry. }\end{array}$ & & 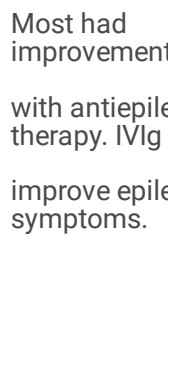 \\
\hline $\begin{array}{l}\text { Cowen et } \\
\text { al., } 2020 \\
\text { [7] }\end{array}$ & Case-report & $\begin{array}{l}\text { Female, } 50 y \\
\text { Evans syndrome }\end{array}$ & $\begin{array}{l}\text { 8y after } \\
\text { neurological } \\
\text { manifestations }\end{array}$ & $\begin{array}{l}\text { Cerebellar ataxia } \\
\text { First diagnosis: } \\
\text { neurosarcoidosis }\end{array}$ & $\begin{array}{l}\text { Normal CSF } \\
\text { Brain MRI: signal } \\
\text { intensity lesion in } \\
\text { right cerebellar } \\
\text { hemisphere with } \\
\text { mass effect }\end{array}$ & $\begin{array}{l}\text { GLILD: } 10 y \\
\text { after } \\
\text { neurological } \\
\text { manifestation } \\
(60 y)\end{array}$ & $\begin{array}{l}\text { Corticosteroi } \\
\text { methotrexate } \\
\text { immunoglob } \\
\text { replacement } \\
\text { (initially } \\
\text { intravenously } \\
\text { subcutaneou }\end{array}$ \\
\hline & & & & & $\begin{array}{l}\text { Brain biopsy: focal } \\
\text { infiltration with } \\
\text { macrophages and } \\
\text { lymphocytes (mainly } \\
\text { T-cells); no } \\
\text { granulomas }\end{array}$ & & $\begin{array}{l}\text { Clinical and i } \\
\text { remission in }\end{array}$ \\
\hline
\end{tabular}




\begin{tabular}{|c|c|c|c|c|c|c|c|}
\hline $\begin{array}{l}\text { Author, } \\
\text { year }\end{array}$ & Type of study & $\begin{array}{l}\text { Gender, age, } \\
\text { clinical history }\end{array}$ & $\begin{array}{l}\text { CVID } \\
\text { diagnosis }\end{array}$ & $\begin{array}{l}\text { Neurological } \\
\text { manifestation }\end{array}$ & $\begin{array}{l}\text { Complementary } \\
\text { study }\end{array}$ & $\begin{array}{l}\text { Other CVID } \\
\text { manifestations }\end{array}$ & $\begin{array}{l}\text { Treatment ar } \\
\text { prognosis }\end{array}$ \\
\hline $\begin{array}{l}\text { Lee et al., } \\
2020 \text { [5] }\end{array}$ & $\begin{array}{l}\text { Cross-sectional } \\
\text { survey (US } \\
\text { Immunodeficiency } \\
\text { Network - registry of } \\
\text { primary } \\
\text { immunodeficiencies) }\end{array}$ & $\begin{array}{l}601 \text { patients, } 29.6 \\
\text { y (range } 0-73), \\
65.9 \% \text { female }\end{array}$ & Yes & $\begin{array}{l}\geq 1 \text { neurologic } \\
\text { symptom in } \\
49.1 \% \\
(601 / 1224) \text { : } \\
\text { - Neuropsychiatric } \\
(53.7 \%) \\
\text { - Headache } \\
\text { (35.6\%) } \\
\text { - Developmental } \\
\text { delay (12.5\%) } \\
\text { - Neuropathy } \\
\text { (10.8\%) } \\
\text { - Seizures (10.7\%) } \\
\text { - Endocrine } \\
\text { disorder (6\%) } \\
\text { - Vertigo (5\%) } \\
\text { - Cerebrovascular } \\
\text { disease (4.7\%) } \\
\text { - changes in } \\
\text { sensory } \\
\text { acuity/function } \\
\text { (4.5\%) } \\
\text { - Sleep disorders } \\
\text { (4.2\%) } \\
\text { - Movement } \\
\text { disorders (3.7\%) } \\
\text { - Cognitive } \\
\text { impairment } \\
\text { (2.8\%) } \\
\text { - Ataxia (2.5\%) } \\
\text { - Altered mental } \\
\text { status (0.5\%) }\end{array}$ & $\begin{array}{l}17(1.4 \%) \text { with } \\
\text { neurologic } \\
\text { autoimmune } \\
\text { diagnoses: cerebral } \\
\text { vasculitis }(n=5) \text { and } \\
\text { demyelinating } \\
\text { disease }(n=5) \text {, brain } \\
\text { granuloma }(n=2) \text {, } \\
\text { autoimmune } \\
\text { sensorineural hearing } \\
\text { loss, autoimmune } \\
\text { encephalitis, } \\
\text { leukoencephalopathy, } \\
\text { myasthenia gravis, } \\
\text { neuritis, } \\
\text { neuromyelitis optica, } \\
\text { and chronic } \\
\text { lymphocytic } \\
\text { inflammation }\end{array}$ & & $\begin{array}{l}\text { Corticosteroi } \\
(n=12) \text {, azatt } \\
(n=2) \text {, abatac } \\
(n=1), \\
\text { hydroxychlor } \\
(n=1) \text {, metho } \\
(n=1), \\
\text { mycophenoli } \\
\text { mofetil }(n=1) \\
\text { sirolimus ( } n=\end{array}$ \\
\hline $\begin{array}{l}\text { Shribman } \\
\text { et al., } \\
2020 \text { [15] }\end{array}$ & Case-report & $\begin{array}{l}\text { Male, } 31 \text { y } \\
\text { Afro-caribbean }\end{array}$ & As a teenager & $\begin{array}{l}\text { Headache: acute- } \\
\text { onset, constant } \\
\text { headache for } 1 \\
\text { month without } \\
\text { fever, meningism, } \\
\text { seizures, nor any } \\
\text { preceding illness } \\
\text { after missing his } \\
\text { treatment with } \\
\text { IVIG } \\
\text { Encephalomyelitis } \\
\text { with retinopathy }\end{array}$ & $\begin{array}{l}\text { CSF: } 68 \text { lymphocytes } \\
\text { and } 1.31 \mathrm{~g} / \mathrm{L} \text { protein } \\
\text { CT brain: hyperdense } \\
\text { lesion in right } \\
\text { callosal body } \\
\text { MRI: restricted } \\
\text { diffusion and } \\
\text { contrast- } \\
\text { enhancement with } \\
\text { several lesions } \\
\text { around the lateral } \\
\text { ventricles; diffuse } \\
\text { intramedullary signal } \\
\text { change within the } \\
\text { entire spinal cord }\end{array}$ & & $\begin{array}{l}\text { Regular IVIg } \\
\text { Symptoms a } \\
\text { abnormalitie } \\
\text { resolved with } \\
\text { steroids } \\
\text { Retinal findir } \\
\text { unchanged }\end{array}$ \\
\hline $\begin{array}{l}\text { Van de } \\
\text { Ven et al., } \\
2020 \text { [16] }\end{array}$ & $\begin{array}{l}\text { Observational and } \\
\text { retrospective study }\end{array}$ & $N=17$ & Yes & $\begin{array}{l}\text { Loss of sensory } \\
\text { or motor function, } \\
\text { headache, or } \\
\text { epilepsy }\end{array}$ & $\begin{array}{l}\text { Contrast-enhancing } \\
\text { lesions in brain and } \\
\text { spinal cord: the most } \\
\text { common findings on } \\
\text { MRI. Molecular } \\
\text { defect in } 8 \text { patients, } \\
\text { including mutations } \\
\text { in CTLA4, NFKB1, } \\
\text { and CECR1 }\end{array}$ & & $\begin{array}{l}\text { CNS involver } \\
\text { usually respc } \\
\text { well to high-c } \\
\text { steroids, but } \\
\text { required mail } \\
\text { therapy to pr } \\
\text { relapse }\end{array}$ \\
\hline $\begin{array}{l}\text { Strunz et } \\
\text { al, } 2021 \\
{[18]}\end{array}$ & $\begin{array}{l}\text { Observational and } \\
\text { retrospective study }\end{array}$ & $N=50$ & Yes & - & - & $\begin{array}{l}12 \%(n=6) \\
\text { radiological } \\
\text { and/or } \\
\text { histological } \\
\text { confirmed } \\
\text { GLILD } \\
\text { diagnosis }\end{array}$ & $\begin{array}{l}\text { Synchronic c } \\
\text { nervous syst } \\
\text { involvement } \\
\text { GLILD-patien } \\
\text { responded to } \\
\text { rituximab tre } \\
\text { All patients s } \\
\text { radiological I } \\
\text { and stabiliza } \\
\text { improvemen } \\
\text { pulmonary ft }\end{array}$ \\
\hline
\end{tabular}


CVID-associated neurological symptoms are considered rare; however, a recent study, including 601 patients selected from the United States Immunodeficiency Network, showed that at least one neurologic condition/symptom was reported in $49.1 \%$ of CVID patients - mostly neuropsychiatric symptoms (53.7\%), followed by headache (35.6\%) and developmental delay (12.5\%) [5]. In another study, Najem et al. [17] reported that, among the CVID patients with granulomatous disease, $68.4 \%$ (13/19) had granulomas involving $\geq 2$ organs, including the CNS, and $31.6 \%$ (6/19) had isolated CNS granulomas. In this study, the most frequent initial neurological symptom was seizures (31.6\%), followed by de novo headache and/or worsening headache (21\%) and vision loss (15.7\%). In our patient, headache was the initially recognized and recurrent manifestation of inflammatory cerebral disease secondary to CVID. However, she had focal temporal epilepsy of undetermined etiology, with her first seizure at 30 years of age, that could be related to neurological manifestations of CVID. Galli et al. [19], in a case series of 5 patients, described an association between CVID and autoimmune-mediated epilepsy and/or atypical epilepsy, including behavioral arrest, alterations in consciousness, and/or amnestic episodes, and less commonly generalized tonic-clonic seizures. A majority of these patients had abnormal imaging findings, most commonly temporal lobe asymmetry, and most improved with antiepileptic therapy, but not with IVIG [19].

Based on Van de Ven's [16] diagnostic and treatment algorithm, we proposed an approach to patients with suspected CVID when neurological manifestations (Table 2). According to Van de Ven's (16) and Farshad et al. [9] the first step is performing a contrast-enhanced MRI of the brain and/or spine [9, 16]. Our patient had a cerebellar lesion with cortico-pial hypersignal in FLAIR, with post-gadolinium T1-enhancement (Figures 1 and 2). Similarly, Van de Ven et al. [16], found that 4 in 9 patients with cerebral contrast-enhancing lesions had also leptomeningeal involvement. In the study of Najem et al. [17], brain mass was the most common radiologic finding followed by leptomeningeal enhancement (10\%). Differential diagnoses included metastases, carcinomatous meningitis, multilocular glioma, high-grade glioma, and lymphoma [16]. Afterward, a lumbar puncture should be done (if not contraindicated) for CSF collection, including leukocyte differential count, protein electrophoresis, glucose, and flow cytometry (if suspected hematological malignancy) [16]. All CVID patients with noninfectious CNS lesions had a nonspecific CSF examination: 75\% of patients had a mild protein elevation and 75\% had a mild mononuclear/lymphocytic pleocytosis [9]. Glucose consumption is not typical of this manifestation, making it more pertinent to exclude an infectious etiology, especially tuberculosis, in our case. Thus, an infectious screening must be performed in all patients, including enteroviruses by PCR (especially when dermatomyositis is suspected), VZV and HSV 1 and 2 PCR, TPPA and VDRL, CMV, EBV, HIV, Borrelia's serology, Interferon-gamma release assay for tuberculosis, Brucella, Immunostaining for Trichinella, hydatica (Cryptococcus and Toxoplasma if CD4 counts are low) [16]. If all of this work-up remains inconclusive, a brain biopsy is recommended [16]. In two case reports similar to ours, with CNS and pulmonary manifestations of CVID, a brain biopsy was performed [4, 7]. However, histological findings were nonspecific, showing focal infiltration of macrophages and lymphocytes, predominantly T-cells [7]. In our case, a brain biopsy was not pursued as the risks outweighed the benefits, and the remaining study was not suggestive of an expansive lesion. 


\section{A. When confirmed/suspected CVID with neurological manifestations}

1. Personal history of increased susceptibility to infection (recurrent infections)

2. and/or family history of antibody deficiency

3. and/or previous history of autoimmune manifestations (frequently autoimmune thrombocytopenia, autoimmune hemolytic anemia, pernicious anemia, and rheumatoid arthritis)

4. and/or presence of granulomatous disease

5. and/or unexplained polyclonal lymphoproliferation

B. Search: immunoglobulin isoforms (IgG, IgA, IgM) - forward to an immunoallergy appointment to exclude other forms of hypogammaglobulinemia

C. Neurological manifestations of CVID (adapted from Van de Ven et al. (16))

C1. Diagnostic evaluation:

- Brain imaging - ideally contrast-enhanced MRI: frequently brain mass lesion, followed by lesions with hypersignal in FLAIR and post-gadolinium T1enhancement

- Cerebrospinal fluid - include leukocyte differential count, glucose, protein electrophoresis, and flow cytometry (if suspected hematological malignancy)

- Infectious screening in all patients: always enteroviruses (PCR), VZV and HSV 1 and 2 (PCR), TPPA and VDRL, CMV, EBV, HIV, Borrelia's serology (Lyme disease), Interferon-gamma release assay from tuberculosis, Brucella, Immunostaining for Trichinella, hydatica (cryptococcus and toxoplasma when CD4 counts are low), JC virus (according to the imaging finding)

- If the all work-up remains inconclusive, a brain biopsy is recommended

- Screening other systemic manifestations (CT TAP, PET)

- Molecular testing for underlying genetic CVID

C2. Treatment

1. High ( $1 \mathrm{mg} / \mathrm{kg} \mathrm{BW} /$ day prednisone equivalent) or medium-dose $(0.5 \mathrm{mg} / \mathrm{kg} \mathrm{BW} /$ day) steroids, tapering within 4 to 6 weeks to $20 \mathrm{mg} / \mathrm{d}$ and then maintenance of $5 \mathrm{mg}$ daily or stopping if responsive

2. In life-threatening or refractory disease: methylprednisolone pulse therapy at a dose of $1 \mathrm{~g}$ for 3-5 days, dexamethasone or additional immunosuppressants (for example cyclophosphamide). Some studies used methylprednisolone iv $1 \mathrm{~g}$ for $3-5$ days + immunoglobulin replacement therapy - if a rapid response is required, use high-dose intravenous immunoglobulin (1-2 g/kg in 5 days)

After 1. and 2. keep low dose of corticosteroids; consider a corticosteroid-sparing maintenance immunosuppressive drug, such as methotrexate, rituximab (good responses in GLILD and associated cerebral manifestation) or infliximab (this last one with low evidence), to reduce the dose of steroid and thus its adverse effects

C3. Follow-up

- At each visit, clinical assessment for neurological symptoms

- Contrast-enhanced MRI brain and/or spine every 2-3 y or when clinically relevant

When diagnosing CVID, it is also important to look for additional organ manifestations [16]. Finally, Van de Ven [16] recommended, if possible, a genetic evaluation for all patients with CNS disease (search for mutations in ADA2, NFKB1, and CTLA4), since targeted therapies are under investigation.

Overall, the diagnosis of CVID-associated neurological manifestations took an average of 2.5 years, 5.75 years after the onset of symptoms [9]. In our case, refractory headache with intracranial hypertension led to the prompt diagnosis of neurological involvement; however, only after lesion recurrence, 5 years later, was associated with CVID. The wide range of differential diagnoses delays the diagnosis, with neurosarcoidosis being the main mimicry of CVID [4, 7, 9,11 , 20]. Although clinical manifestations can be similar, the key difference between CVID and sarcoidosis are the low immunoglobulin levels in CVID and the normal or high immunoglobulin levels in sarcoidosis [9]. Note that ACE levels do not discriminate CVID and sarcoidosis [8]

Pulmonary involvement is more common than neurological involvement in patients with CVID [7]. GLILD is a specific subtype of interstitial lung disease associated with CVID, characterized by granulomas and/or benign diffuse lymphocytic infiltrates in the pulmonary parenchyma [21]. This disease is often unrecognized and misdiagnosed by physicians [21]; however, it represents a common organ manifestation of CVID, with a prevalence of $8-22 \%$. It is the type of lung involvement in CVID with the worst prognosis [7, 18]. Although a thoracic CT is required to support its diagnosis, there is no specific radiological feature sufficient to confirm this diagnosis [7]. GLILD can be detected on lung biopsies many years before the CVID diagnosis [2]: noncaseating granulomas and lymphocytic infiltrates (as happened in our patient) were consistent features; interstitial fibrosis with architectural remodeling is also frequent [21]. Thus, exclusion of other potential pulmonary pathologies, such as organizing pneumonia, mycobacterial infection, malignancy, and lymphoma, is a key consideration in the diagnosis of GLILD, and it is frequent to consider these patients as having uncomplicated sarcoidosis [8, 21]. There are clues, although not definitive, for the distinction of these two pathologies: 1) concurrent autoimmune disease, such as autoimmune cytopenias, occurs much less frequently in sarcoidosis than CVID [20]; 2) sarcoidosis is more commonly associated with uveitis and skin disease [20]; 3) scattered nodules with areas of consolidation and ground glass, prominent on the lower zones, and numerous bronchiectasis, and compatible biopsy features favored a diagnosis of GLILD instead of sarcoidosis; 4) GLILD patients usually also exhibited almost normal physiologic function (some patients could have a restrictive pattern of pulmonary function tests) $[7,8]$. 
To date, there is no established standard of care for the treatment of patients with CVID, particularly with GLILD or neurological complications [16]. In patients with CVID with neurological or other associated symptoms, and/or evidence of a decline in lung function, after exclusion of infectious and malignant causes, treatment with steroids seems to be safe and effective control neurological manifestations [16]. IVlg replacement is the recommended therapy for symptomatic CVID patients, and it seems to be relevant in the prevention and treatment of GLILD complications, as was evident in our case.

According to Van de Ven et al. recommendations [16], in the acute phase, high or medium-dose steroids (depending on the case's severity) should be used, tapering within 4-6 weeks to $20 \mathrm{mg} /$ day, subsequently maintaining a low dose of corticosteroid therapy or stopping if responsive. In life-threatening or refractory diseases, most patients acutely received high-dose intravenous glucocorticoids, specifically, methylprednisolone at a dose of $1 \mathrm{~g}$ initially for $3-5$ days, as well as immunoglobulin replacement therapy $[2,4,7,9-12,14,16]$ - if a rapid response is required, authors use high-dose intravenous immunoglobulin (1-2 g/kg in 5 days) [16]. Only in one case IVIg was used without concomitant corticosteroid therapy [15]. Some authors consider a corticosteroid-sparing maintenance immunosuppressive drug, such as methotrexate [5, 7, 9], infliximab [11] or rituximab [4], to reduce the dose of steroid and thus its adverse effects, with good results. In particular, some recent studies have shown that rituximab in combination with prednisolone represents an effective therapy for GLILD, also achieving clinical and MRI-morphological regression of the neurological manifestation of CVID [4, 18, 22]. Even though the major part of its immunomodulatory effects are accountable to B-cell depletion, rituximab may also target both helper and cytotoxic T-cells, increasing the T regulatory cells and normalizing of Th1/Th2 ratio [22]. These effects support its use as a second line in autoimmune and lymphoproliferative complications of CVID [22]. In relation to infliximab, it had mixed results in CVID patients with neurological manifestations [18]. Reports suggest that a synergistic effect of intravenous immunoglobulin and infliximab improves neuroimaging as well as systemic features such as fever, night sweats and lymphadenopathy, whilst having no clinical effect on neurological deficits [18].

In case of detection of a specific mutation, studies report better results with targeted therapy: patients with DADA2 mutations may be candidates for anti-TNF therapy, with CTLA-4 or LRBA mutations for abatacept, those with a gain of function mutations of GOF-PIK3CD or loss of function LOF-PIK3R1 (APDS 1 and 2) may improve with mTOR inhibitors, like rapamycin, or newer agents such as Idelalisib [1].

For the follow-up study, Van de Ven et al. [16] recommend performing a contrast-enhancement MRI at least every 2-3 years, or if relapse is suspected. In the described cases of neurological manifestations of CVID, patients had generally a good prognosis, with clinical and imaging remission in the majority within three months, as long as the therapy with steroids and immunoglobulins was maintained [9]. However, these patients have a high relapse rate after treatment interruption [16], as happened with our patient. Ameratunga et al. [23] had recently described a disease severity score for CVID and CVID-like disorders (CDSS). The CDSS focuses on cumulative damage caused by CVID, divided into three categories - mild, moderate, and severe. Mortality is increased in patients with functional or structural lung disease or hepatitis [23]. In our patient, damage to the CNS is unlikely to result in mild complications, most are likely to be lifethreatening or have the potential to cause severe disability [23]. Our patient scored 10 (severe disease) in CNS manifestations, 5 in pulmonary parameters (mild GLILD, mild bronchiectasis), and 1 for asymptomatic splenomegaly, out of a total of 16 in the CDSS severity score.

In conclusion, a CVID-associated neurological disorder is a rare but often severe manifestation. Its diagnosis is based on a combination of compatible clinical, radiological, and histological features; exclusion of other pathologies, such as malignancy, lymphoma, mycobacterial disease, and sarcoidosis, should be carried out in all cases. Symptomatic CNS disease appears to respond rapidly to treatment with high-dose steroids, but relapses occur in a considerable number of patients if treatment is discontinued; therefore, follow-up is of utmost importance.

\section{Declarations}

Funding: The authors declare that no funds, grants, or other support were received during the preparation of this manuscript.

Conflicts of interest/Competing interests: The authors declare that they have no conflict of interest. The authors have no relevant financial or non-financial interests to disclose.

Availability of data and material: All data generated or analysed during this study are included in this published article.

Code availability: not applicable.

Authors' contributions: All authors contributed to the study conception and design. BM, JM and MJP made substantial contributions to conception and study design. BM and JM worked on acquisition of data, its analysis and interpretation. BM and JM wrote the first draft. JG, AC, NM, ASP, FC and JTC critically revised and finally approved the version to be published. All authors fully complied with and approved the version to be published.

Ethics approval: This work does not require ethics approval.

Consent to participate: Written informed consent was obtained from the parent.

Consent for publication: The participant has consented to the submission of the case report to the journal.

\section{References}

1. Ameratunga R, Woon ST. Perspective: Evolving Concepts in the Diagnosis and Understanding of Common Variable Immunodeficiency Disorders (CVID). Clin Rev Allergy Immunol. 2020;59(1):109-21. DOI:10.1007/s12016-019-08765-6.

2. Abati E, Faravelli I, Magri F, Govoni A, Velardo D, Gagliardi D, et al. Central nervous system involvement in common variable immunodeficiency: A case of acute unilateral optic neuritis in a 26 -year-old Italian Patient. Front Neurol. 2018;9:1-7. DOI:10.3389/fneur.2018.01031.

Page $10 / 13$ 
3. Selenius JS, Martelius T, Pikkarainen S, Siitonen S, Mattila E, Pietikäinen R, et al. Unexpectedly high prevalence of common variable immunodeficiency in Finland. Front Immunol. 2017;8:1-10. DOI:10.3389/fimmu.2017.01190.

4. Nguyen JTU, Green A, Wilson MR, DeRisi JL, Gundling K. Neurologic Complications of Common Variable Immunodeficiency. J Clin Immunol. 2016;36(8):793-800. DOI:10.1007/s10875-016-0336-8.

5. Lee M, Nguyen J, Fuleihan R, Gundling K. Neurologic Conditions and Symptoms Reported Among Common Variable Immunodeficiency Patients in the USIDNET. J Clin Immunol. 2020;40(8):1181-3. DOI:10.1007/s10875-020-00861-z.

6. Edgar DES. ESID Registry - Working definitions for clinical diagnosis of PID [Internet]. 2016. Available from: https://esid.org/Working-Parties/RegistryWorking-Party/Diagnosis-criteria.

7. Cowen JE, Stevenson J, Paravasthu M, Darroch J, Jacob A, Tueger S, et al. Common variable immunodeficiency with granulomatous-lymphocytic interstitial lung disease and preceding neurological involvement: A case-report. BMC Pulm Med. 2020;20(1):1-8. DOI:10.1186/s12890-020-01231-6.

8. Boursiquot JN, Gérard L, Malphettes M, Fieschi C, Galicier L, Boutboul D, et al. Granulomatous disease in CVID: Retrospective analysis of clinical characteristics and treatment efficacy in a cohort of 59 patients. J Clin Immunol. 2013;33(1):84-95. DOI:10.1007/s10875-012-9778-9.

9. Farshad S, Figueroa Rodriguez F, Halalau A, Skender J, Rasmussen C, Pevzner M. The Central Nervous System Effects and Mimicry of Common Variable Immunodeficiency (CVID): A Case Report with Literature Review. Case Rep Rheumatol. 2019;2019:1-6. D0I:10.1155/2019/7623643.

10. Jabbari E, Marshall CR, Longhurst $\mathrm{H}$, Sylvester R. Longitudinally extensive transverse myelitis: A rare association with common variable immunodeficiency. Pract Neurol. 2015;15(1):49-52. DOI:10.1136/practneurol-2014-000953.

11. Kumar N, Hagan JB, Abraham RS, Aksamit AJ. Common variable immunodeficiency-associated myelitis: Report of treatment with infliximab. J Neurol. 2008;255(11):1821-4. DOI:10.1007/s00415-008-0898-3.

12. Danieli MG, Pettinari L, Marinangeli L, Logullo F. (2012). Recurrent myelitis in common variable immunodeficiency successfully managed with high-dose subcutaneous immunoglobulin. BMJ Case Rep. 4-7. D0I:10.1136/bcr-01-2012-5637.

13. Misbah SA, Spickett GP, Esiri MM, Hughes JT, Matthews WB, Thompson RA, et al. Recurrent intra-cranial granulomata presenting as space-occupying lesions in a patient with common variable immunodeficiency. Postgrad Med J. 1992;68(799):359-62. D0I:10.1136/pgmj.68.799.359.

14. Sempere AP, Tahoces M, Palao-Duarte S, Garcia-Perez A. Bilateral optic neuritis in a 26-year-old man with common variable immunodeficiency: A case report. J Med Case Rep. 2011;5:5-6. DOI:10.1186/1752-1947-5-319.

15. Shribman SE, Katanga J, Ali N, Hayman GR, Bridges LR, Habibi MS, et al. Encephalomyelitis with Retinopathy in Common Variable Immunodeficiency (CVID). Neuro-Ophthalmology. 2020;44(1):38-40. DOI:10.1080/01658107.2018.1542008.

16. van de Ven A, Mader I, Wolff D, Goldacker S, Fuhrer H, Rauer S, et al. Structural Noninfectious Manifestations of the Central Nervous System in Common Variable Immunodeficiency Disorders. J Allergy Clin Immunol Pract. 2020;8(3):1047-62.e6. DOI:10.1016/j.jaip.2019.11.039.

17. Najem CE, Springer J, Prayson R, Culver DA, Fernandez J, Tavee J, et al. Intra cranial granulomatous disease in common variable immunodeficiency: Case series and review of the literature. Semin Arthritis Rheum. 2018;47(6):890-6. DOI:10.1016/j.semarthrit.2017.10.014.

18. Strunz P-P, Fröhlich M, Gernert M, Schwaneck EC, Nagler L-K, Kroiss A, et al. Rituximab for the Treatment of Common Variable Immunodeficiency (CVID) with Pulmonary and Central Nervous System Involvement. Open Rheumatol J. 2021;15(1):9-15. DOI:10.2174/1874312902115010009.

19. Galli J, Kresser J, Klein J, Gundlapalli A, Graff-Radford J, Greenlee J SC (2019). Atypical Epilepsy in Common Variable Immunodeficiency: A Single Institution Case Series (P1.2-044). Neurology. 92(15 Supplement).

20. Narula N, lannuzzi M. Sarcoidosis: Pitfalls and Challenging Mimickers. Front Med. 2021;7(January):1-15. DOI:10.3389/fmed.2020.594275.

21. Cinetto F, Scarpa R, Carrabba M, Firinu D, Lougaris V, Buso H, et al. Granulomatous Lymphocytic Interstitial Lung Disease (GLILD) in Common Variable Immunodeficiency (CVID): A Multicenter Retrospective Study of Patients From Italian PID Referral Centers. Front Immunol. 2021;12:1-10. DOI:10.3389/fimmu.2021.627423.

22. Pecoraro A, Crescenzi L, Galdiero MR, Marone G, Rivellese F, Rossi FW, et al. Immunosuppressive therapy with rituximab in common variable immunodeficiency. Clin Mol Allergy. 2019;17(1):1-11. DOI:10.1186/s12948-019-0113-3.

23. Ameratunga R. Assessing Disease Severity in Common Variable Immunodeficiency Disorders (CVID) and CVID-Like Disorders. Front Immunol. 2018;9:2130,1-8. DOI:10.3389/fimmu.2018.02130.

\section{Figures}



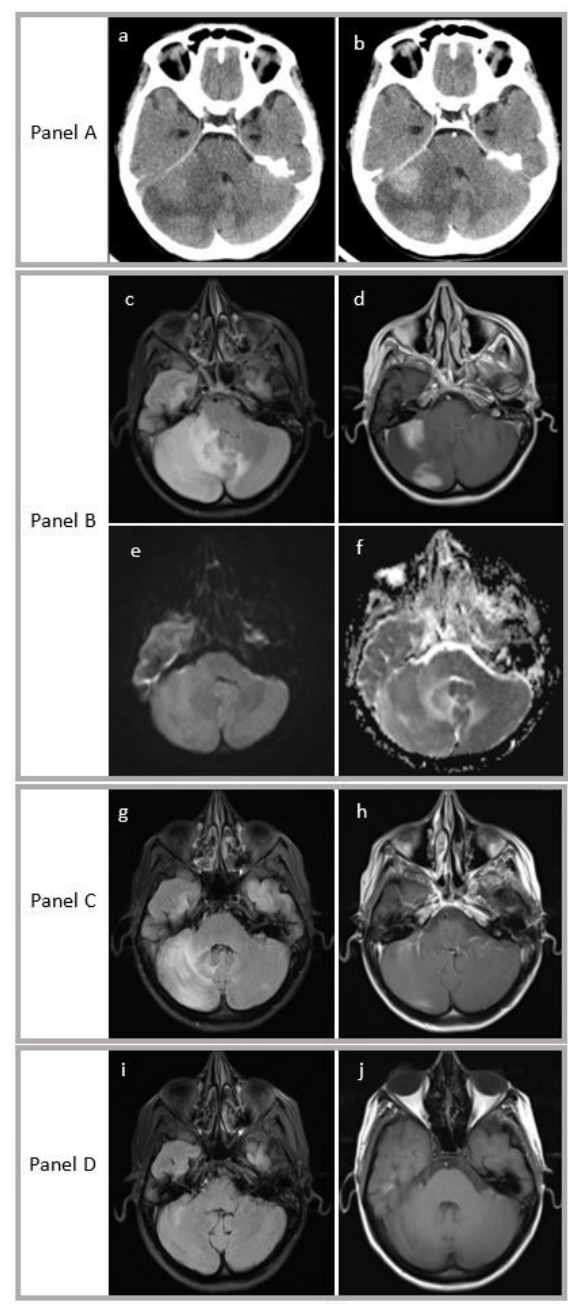

\section{Figure 1}

Brain imaging. Panel A: non-enhanced (a) and contrast-enhanced (b) Brain CT (April 27/2015) showing two contrast-enhanced lesions in the right cerebellar hemisphere, perilesional edema, obstructive hydrocephalus, and brainstem compression. Panel B: Brain MRI (April 28/2015) showing high signal in both cerebellar hemispheres (right predominance), associated with edema, cerebellar folia effacement, left fourth ventricle compression, superior cerebellar vermis deviation, dehiscence of the cerebellar tonsils (right predominance), and enlargement of the supratentorial ventricular system, and the cortico-subcortical region of the right inferior medial temporal lobe. (c) axial FLAIR, an arrow with a corresponding abnormal focal area of enhancement shown on the (d) axial T1 post-gadolinium images. These lesions had no restriction to diffusion (e and f). Panel C: MRI while the patient was on treatment (May/2015, one month after) showing reduction in the corticopial enhancement areas, without ventricular dilatation, in (g) axial FLAIR and (h) T1 post-gadolinium. Panel D: One year after, 2016, MRI also showing control of brain disease in (i) axial FLAIR and (j) T1 post-gadolinium. 

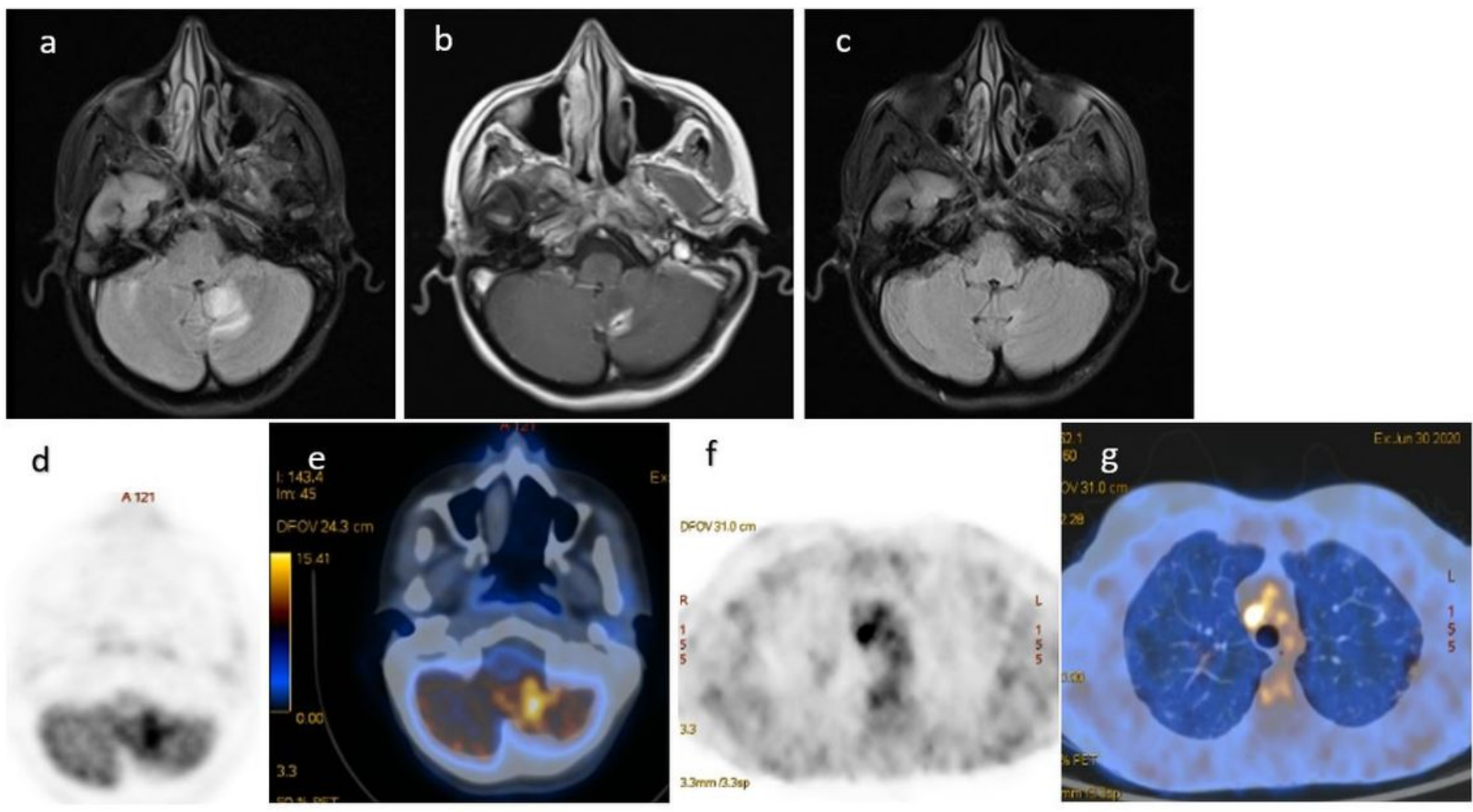

\section{Figure 2}

Repetition of the cerebral and pulmonary study in suspected CVID manifestations recurrence. Brain MRI (June 2020) showing a new corticopial enhancement mainly in the inferior aspect of left cerebellar hemisphere, with pachymeningeal enhancement in the cerebellar tent, in (a) axial FLAIR, (b) T1 post-gadolinium, without restriction to diffusion (not shown). Image (c) showing brain MRI (august 2020) with the return of Ig replacement and high-dose corticosteroid therapy. PET/CT study (June 2020) showing ( $d$ and e) increased FDG-F18 uptake and heterogeneous distribution of cerebellar FDG-F18, and ( $f$ and g) irregular densifications in both lungs and mediastinal-hilar bilateral ganglion formations. 